\title{
The Expression and Biological Function of DKK1 in Oral Squamous Cell Carcinomas by Bioinformatics Analysis
}

\author{
Huijie Yü, Tianhua Li", Xuemei Mao* \\ Department of Stomatology, The People's Hospital of \\ Dongying, Shandong, China \\ "Contribute Equally to this Work \\ *Corresponding author: Xuemei Mao, Department \\ of Stomatology, The People's Hospital of Dongying, \\ Shandong, China
}

Received: March 13, 2021; Accepted: April 21, 2021; Published: April 28, 2021

\begin{abstract}
Objective: To clear the expression of transcription factor Dickkopf-1 (DKK1) in Oral Squamous Cell Carcinoma (OSCC) using the method of bioinformatics analysis. And to clarify the relationship between the expression of DKK1 and the clinicopathological characteristics of OSCC using the method of molecular biology and cytobiology, in order to determine the early diagnosis and significance of OSCC according to the marker of DKK1.
\end{abstract}

Methods: In this study, the expression level of DKK1 in OSCC tissues was analyzed using GEPIA and TCGA databases, and then verified in vitro by qRTPCR and Western-blot analysis. The correlation between DKK1 gene expression and the clinical pathological parameters of OSCC, and also the impact of DKK1 on prognosis were determined using the LinkedOmics database. In addition, DKK1 was knocked down by RNA interference in SCC-4 and SCC-25 OSCC cell lines and the proliferation ability of OSCC cells was assessed by MTT assay.

Results: High expression of DKK1 in OSCC is positively correlated with the pathological grade and T stage of OSCC. According to the TCGA results, DKK1 mRNA was highly expressed and it is related to the overall survival rate of OSCC. In addition, the expression level of both DKK1mRNA and protein were significantly raised in the cell line SCC-25 and SCC-4. Furthermore, MTT analysis showed that DKK1 knockdown resulted in reduced proliferation of OSCC cells.

Conclusions: TCGA database analysis showed that DKK1 was highly expressed in OSCC, and it is closely correlated to the pathological parameters of OSCC, which will provide important theoretical guidance for the subsequent study of oral squamous cell carcinoma.

Keywords: DKK1; Oral Squamous Cell Carcinomas (OSCC); Bioinformatics analysis; Biomarker

\section{Introduction}

Oral Squamous Cell Carcinomas (OSCC) is one of the most common oral malignant tumors, accounting for $90 \%$ of the incidence and ranking the $6^{\text {th }}$ place among systemic tumors [1]. The etiology of OSCC is complex. At present, many scholars believe that the disrupted balance between oncogene activation and tumor suppressor gene suppression may be one of the important causes of OSCC [2,3]. It has brought difficulties to the clinical treatment of OSCC because of the insidious onset, highly malignancy, rapid progression, high rate of relapse, and hardly to diagnose in the early stage $[4,5]$. Therefore, the exploration of oncogenes closely related to OSCC is expected to provide a new direction for tumor gene-targeted therapy.

Dickkopf-1 (DKK1) is part of the DKK proteins family. The secreted proteins family shares a similar conserved cysteine domain and inhibits the Wnt/ $\beta$-catenin pathway [6,7]. DKK1 participates in apoptosis through the $\mathrm{Wnt} / \beta$-catenin signaling pathway [8]. DKK1 disorder is associated with the pathogenesis of a great many cancers. There is much evidence showed that DKK1 upregulation contributes to the development of many cancers such as prostatic cancer and nonsmall cell lung carcinoma [9-13]. On the other hand, DKK1 has been shown to be under-expressed in colorectal cancer and gastric cancer
[14]. In Chronic Lymphoblastic Leukemia (CLL), DKK1 is expressed at normal level, but does not affect the Wnt/ $\beta$-catenin pathway. In multiple myeloma, DKK1 has been proved to be a stress response gene involved in the JNK pathway $[15,16]$. As mentioned above, these studies have shown that the activity and expression level of DKK1 are different in different cancers. However, the role of DKK1 in OSCC is still unclear and needs to be further investigated.

In this study, the role of DKK1 in oral squamous cell carcinoma was analyzed through the database website and verified by Quantitative real-time PCR and Western-blot analysis, so as to provide a theoretical basis for determining its regulatory mechanism and whether it can be used as a predictor of prognosis in patients with OSCC.

\section{Materials and Methods}

\section{GEPIA database}

GEPIA (Gene Expression Profiling Interactive Analysis) is a database for dynamic analysis of gene expression data, developed by Beijing university online database (http://gepia.cancer-pku.cn) combined with TCGA GTEx and analyze Gene Expression in different tumors in the database. In this study, the expression of DKK1 and its 
correlation with pathological analysis were analyzed in OSCC tissues and normal tissues.

\section{LinkedOmics database}

LinkedOmics database is third-party online tools for analyzing TCGA database (http://linkedomics.org/login.php). In this study, the website was used to analyze the RNAseq data in TCGA to understand the relationship between the mRNA level of DKK1 and the clinicopathological characteristics of OSCC. Using this site to analyze data requires only 5 steps: (1) Select the type of tumor to be analyzed, "oral squamous cell carcinoma" was selected in this present study; (2) Select the specific RNAseq data of oral squamous cell carcinoma; (3) Input the name of the gene to be analyzed, and fill in DKK1 here;(4) Select the data content of joint analysis, and "Clinical data" is selected in this step;(5) Select statistical method and non-parametric test. After submitting, wait for the analysis result, and click the corresponding option to view.

\section{String-DB database}

String database (https://string-db.org/) is a database for analyzing the interaction between genes or proteins, including direct physical interaction between proteins and indirect functional correlation between proteins. In addition to experimental data, PubMed abstracts, and other database data, it also contains predicted results using the bioinformatics methods. In this study, "DKK1" was input, "human" was selected for species, "Medium 0.4" for confidence, and 20 for maximum number interaction.

\section{Quantitative real-time PCR}

Quantitative Real-Time PCR (QRT-PCR) was used to inoculate HOK cells from normal oral epithelial cells, SCC-25 cells from oral squamous cell lines and SCC-4 cells into 6-well plates at a density of $1.5 \times 10^{5}$ cells per well (grown in RPMI 1640 medium at $37^{\circ} \mathrm{C}$ under $\left.5 \% \mathrm{CO}_{2}\right)$. After $24 \mathrm{~h}$, the mRNA of cells was extracted by Trizol (Invitrogen Carlsbad, USA) according to the manufacturer's instructions. RNA was quantitated with a NanoDrop spectrophotometer (Thermo, USA). According to the manufacturer's instructions, the mRNA samples were reverse transcribed into cDNA using a commercial reverse transcription system (Thermo scientific, USA). The relative PCR quantification was performed using a commercial RT-PCR Kit (TaKaRa, Japan). Using the $-\Delta \Delta \mathrm{CT}$ method, the gene expression data were normalized to the amount of glyceraldehyde-3-phosphate dehydrogenase (GAPDH) mRNA. Primer sequences of DKK1: forward: AACGCTATCAAGAACCTGC, reverse: GATGACCGGAGACAAACA, target fragment of 460bp. Primer sequences of GAPDH: forward: GGGAGCCAAAAGGGTCATCATCTC, reverse: CCATGCCAGTGAGCTTCCCGTTC, target fragment of 353bp. All the primers used in this study were synthesized by AUGCT (Beijing, China).

\section{Western-blot analysis}

Total proteins of the cells were dissolved in lysis buffer and extracted following the manufacturer's protocol (Beyotime Institute of Biotechnology, Haimen, China). The concentration of the target proteins was determined using the Bicinchoninic Acid (BCA) protein assay kit (Thermo Fisher Scientific, Wilmington, DE, USA). Equal amounts of protein were separated by SDS-PAGE using 10\% horizontal gels. And then the proteins were transferred onto a polyvinylidene difluoride membrane (EMD Millipore Corp., Billerica, MA) in a wet blotting system. Membranes were blocked for $1 \mathrm{~h}$ at room temperature and then incubated with the specific primary antibodies overnight at $4^{\circ} \mathrm{C}$. After being washed, membranes were incubated with a secondary Horseradish Peroxidase (HRP)-coupled antibody and processed for enhanced chemiluminescence detection using Immobilon HRP substrate (EMD Millipore Corp., Billerica, MA). Signals were visualized and analyzed on a VisionWorks LS (UVP, BioSpectrum Imaging System, USA). The integrated density of the bands came from the different proteins was quantified using ImageJ Software (National Institute of Health, Bethesda, MD, USA). The ratio of the integrated density of the target protein to that of GAPDH as the loading control was calculated to represent the expression level of protein. Antibodies were used as follows: antiDKK1-1 diluted 1:1,000, and anti-GAPDH diluted 1:1,000, which applied from Santa Cruz (Santa Cruz Biotechnology Inc., CA).

\section{MTT assay}

SCC- 4 and SCC- 25 cells were both inoculated into 96-well plates at 3,000 cells per $100 \mu$ culture media per well, and transfected the next day. A total of $10 \mu \mathrm{l}$ MTT reagent $(5 \mathrm{mg} / \mathrm{ml})$ was added to each well at each time point after the transfection (24, 48 and $72 \mathrm{~h}$ respectively), and $150 \mu$ dimethyl sulfoxide was added after $4 \mathrm{~h}$. The FLUOstar OPTIMA microplate reader (BMG) was used to measure the cell viability of the sample at $490 \mathrm{~nm}$. Following transfection with si-DKK1 or its control, the cells were further cultivated for an additional 1-3 days. Each experiment contained three replicates and was repeated at least twice.

\section{Statistical analysis}

All the experiments were performed at least three times in the same environment, and data were all expressed as means \pm S.E.M. Significance was established with the SPSS 22.0 software (IBM, USA). Student's $t$-test and ANOVA analysis were used if the quantitative data between groups show normal distribution. If not consistent with the normal distribution, using the Wilcoxon-Mann-Whitney test, and a $P$ value $<0.05$ was considered as significant.

\section{Results}

\section{DKK1 mRNA was highly expressed in OSCC and normal oral epithelial tissues}

The GEPIA database was used to analyze the expression of DKK1 in different tumors. The results showed that the expression level of DKK1 was significantly increased in most tumors, such as esophageal cancer, squamous cell carcinoma, pancreatic cancer, including head and neck squamous cell carcinoma (Figure 1). The expression of DKK1 mRNA in OSCC $(\mathrm{n}=519)$ and normal head and neck epithelial tissue $(n=44)$ was further analyzed. And the results showed that compared with normal head and neck epithelial tissue, DKK1 mRNA was expressed extremely high in OSCC. The difference was statistically significant $(\mathrm{P}<0.05)$ and was shown in Figure 2. These results suggest that DKK1 may play an important role in the occurrence and development of OSCC.

Correlation between DKK1 mRNA expression and the clinicopathological characteristics of OSCC

Given that the mRNA expression of DKK1 exhibited high level 


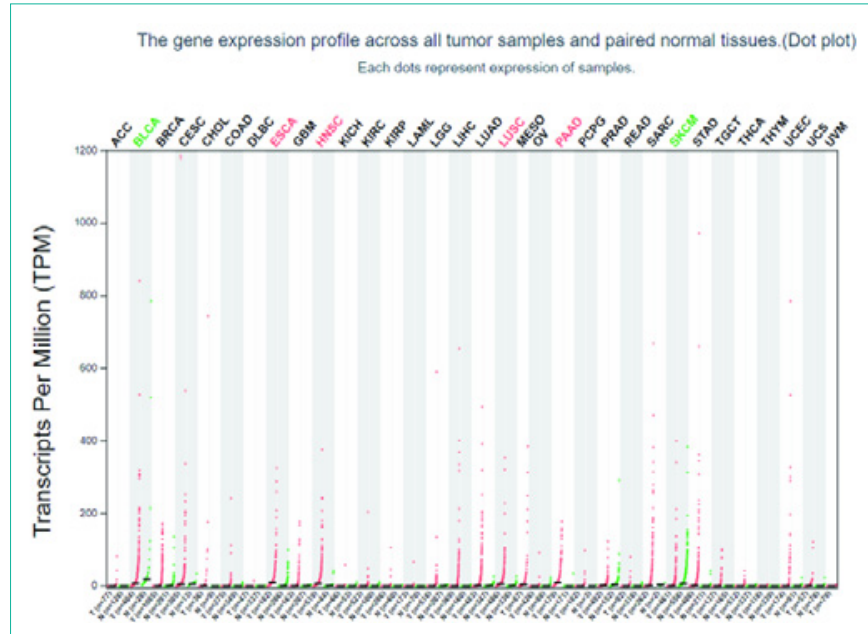

Figure 1: DKK1 expression in different kind of tumors. The expression levels of DKK1 in different tumors, and the type of tumor with red at the top indicates that DKK1 is highly expressed in this tumor compared with normal tissues.

in OSCC compared to the normal tissues, we studied the correlation between DKK1 mRNA expression and the clinicopathological features of OSCC using the software of LinkedOmics. And it was found that in terms of pathological stage $(\mathrm{P}=0.004537, \mathrm{n}=448)$ and $\mathrm{T}$ stage $(\mathrm{P}=0.0006259, \mathrm{n}=458)$, the differences between the two groups that DKK1 expressed high and low were statistically significant, as shown in Figure 3.The results above suggest that the high expression of DKK1 is related to the malignant degree and disease progression of OSCC.

\section{Relationship between DKK1 mRNA expression and prognosis of OSCC}

Moreover, to determine the relationship between DKK1 mRNA expression and the prognosis of OSCC, we used GEPIA database to analyze the correlation between DKK1 mRNA level and the survival

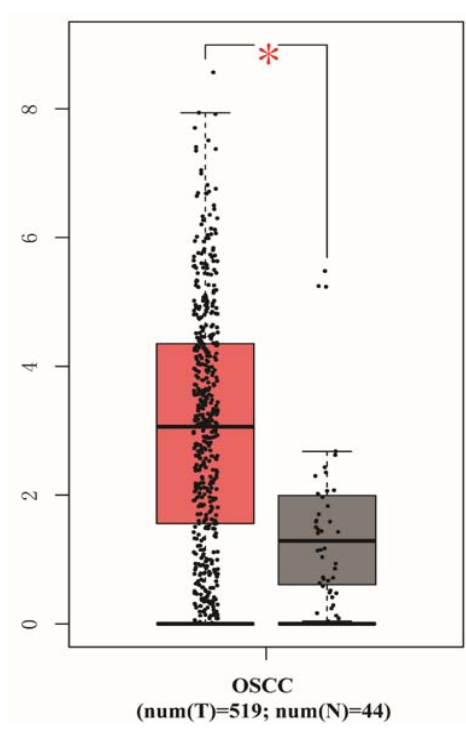

Figure 2: DKK1 mRNA expression in OSCC and normal oral epithelial tissues. qRT-PCR analysis showed that DKK1 mRNA expression was significantly higher in OSCC $(n=519)$ compared to that in normal oral epithelial tissues $(n=44)\left({ }^{*} P<0.05\right.$ from the source file). rate of OSCC patients. The results indicated that the expression level of DKK1 was negatively correlated with the overall survival rate of OSCC patients. The overall survival rate of patients with high DKK1 expression was significantly lower than that of patients with low DKK1 expression $(\mathrm{P}<0.001)$ as shown in Figure 4. The results above suggest that the high expression of DKK1 strongly affect the survival of HNCS patients, and is related to the early postoperative death and early recurrence of OSCC patients.

\section{QRT-PCR and Western-blot analysis of the DKK1expression in OSCC}

Further, in order to verify the high expression of DKK1 in OSCC by cytobilogy, we screened SCC- 25 and SCC- 4 as two oral squamous cell lines, then used QRT-PCR and Western-blot analysis to determine the expression of DKK1 in the above cell lines. As shown in Figure $5 \mathrm{~A}$, DKK1 mRNA was highly expressed in SCC-25 and SCC-4 of oral squamous cell lines compared with HOK of normal oral epithelial cell lines, and the difference was statistically significant $(\mathrm{P}<0.05)$.

To extend our observations at the genetic level, we characterized the expression level of DKK1 protein in different cell lines using western-blot. Compared with normal oral epithelial cell lines HOK, western-blot analysis demonstrated that the protein expression
$\mathbf{A}$

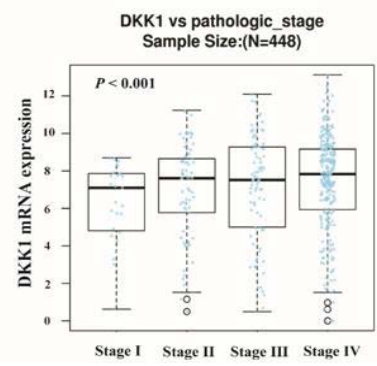

B

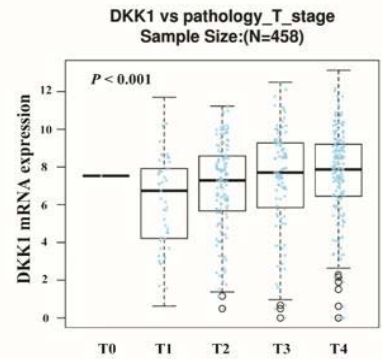

Figure 3: Correlation between DKK1 mRNA expression and clinicopathological features of OSCC. Association of DKK1 mRNA level and pathological features in OSCC. (A) The pathologic stage of DKK1 mRNA level $(P=4,537 e-04$ $n=448)$ and $(B) T$ stage of DKK1 mRNA level $(P=6.259 e-04, n=458)$. Box plots were produced using Linked Omics (http://www.linkedomics.org/login. php), and statistically tested using the Kruskal-Wallis test.

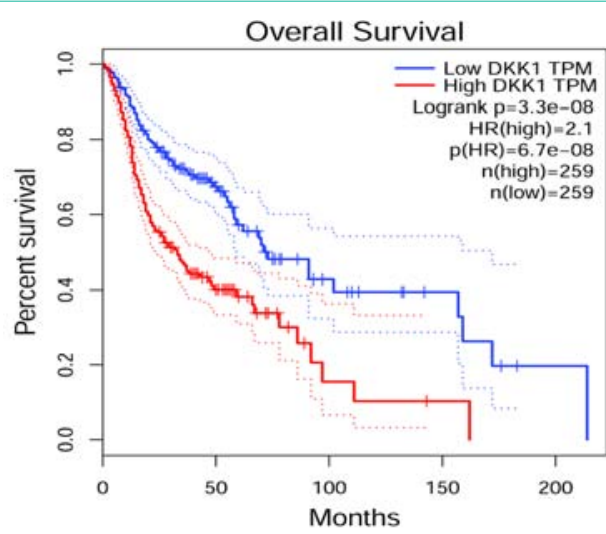

Figure 4: Overall survival of OSCC patients related to different DKK1 status (mRNA level) based on TCGA data Kaplan-Meier analysis indicated that the expression of DKK1 was negatively correlated with the overall survival rate of all 518 patients with OSCC $\left({ }^{* * *} \mathrm{P}<0.001\right.$ from the source file). 


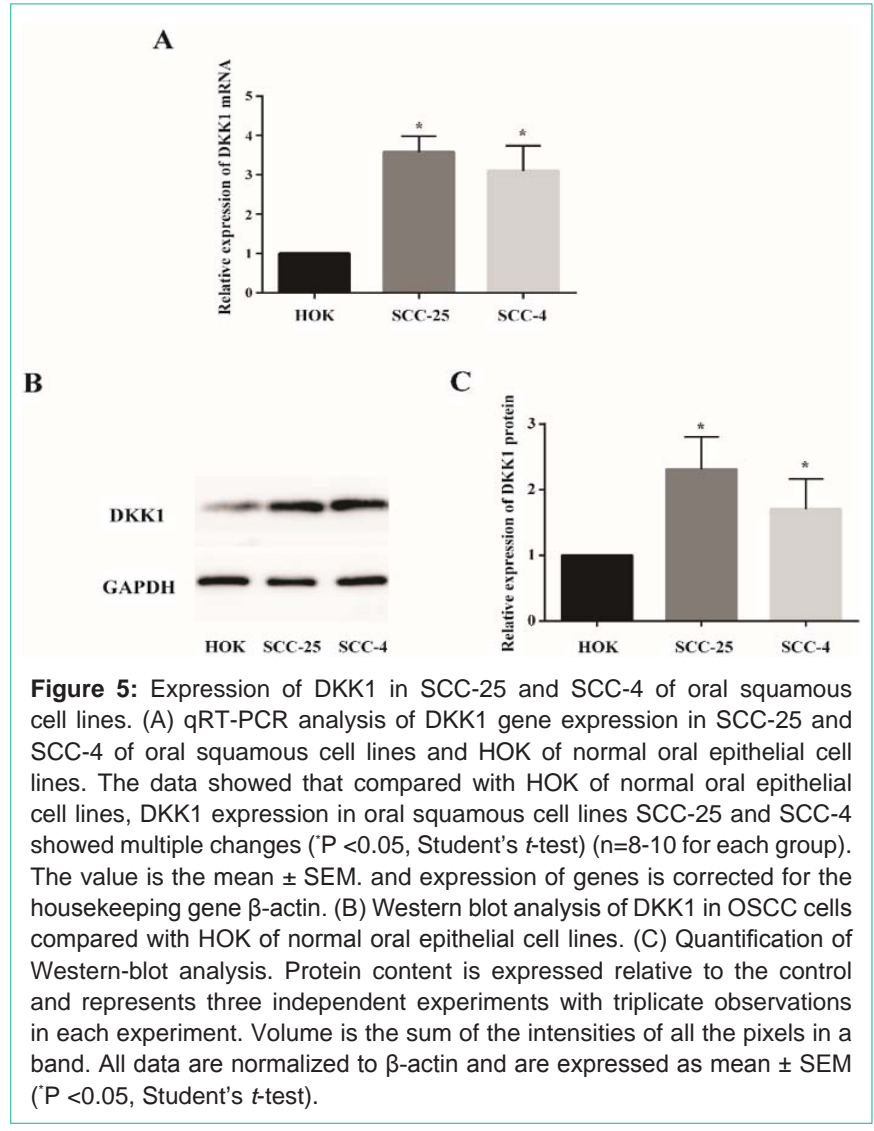

in SCC-25 and SCC-4 cell lines was increased obviously, which is consistent with the results of the gene expression (Figure 5B). And the difference was statistically significant $(\mathrm{P}<0.05)$ (Figure 5C).

\section{Suppressing DKK1 expression inhibited the growth of OSCC cells}

To examine the effects of DKK1 on the growth of OSCC cells, we designed the siRNA for DKK1 (si-DKK1), which induced $60 \%$ decrease of DKK1 expression both at the protein and RNA levels in OSCC cells. MTT assay showed that downregulation of DKK1 suppression of the SCC- 4 and SCC-25 cells growth at $48 \mathrm{~h}$ and $72 \mathrm{~h}$. This finding suggested that DKK1 may promote the proliferation of OSCC cells.

\section{Discussion}

OSCC is one of the most common tumors in the world at present, and its incidence has been younger in the past 30 years, which is threatening the survival and health of human being $[17,18]$. Although the current treatment plan for OSCC is constantly improving, unfortunately the OSCC patients still have a low 5-year survival rate less than $50 \%$. Therefore, it is urgent to understand the occurrence and development of OSCC and discover effective gene-targeted treatment methods [19].

The DKK1 is frequently overexpressed in prostate cancer, gastric cancer, colorectal cancer, non-small cell lung cancer and breast cancer [20-23]. It is suggesting that aberrant DKK1 expression contribute to progression of malignancies [24]. However, there are few reports to evaluate the expression of DKK1 in oral squamous cell carcinoma

\section{A}

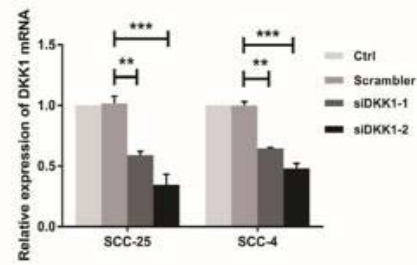

B

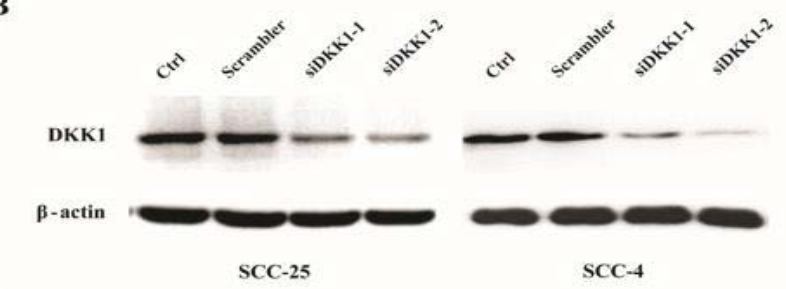

C
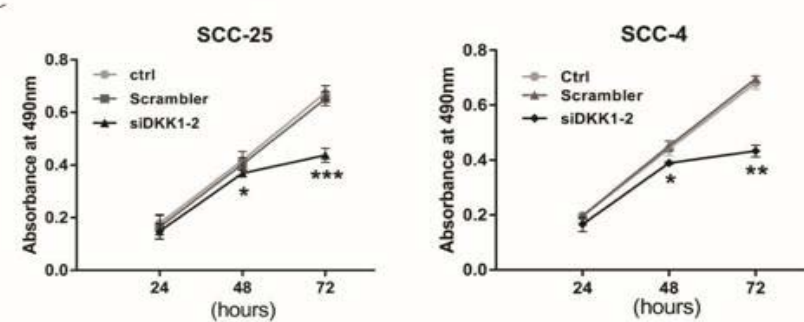

Figure 6: Silencing of DKK1 reduces OSCC cells growth. (A) qRT-PCR and Western blot analysis of DKK1 expression in SCC-25 and SCC-4 cells by silencing of DKK1 using si-DKK1; (B) Proliferation of OSCC cells was measured by MTT assay at 24,48 and $72 \mathrm{~h}$ post-transfection with siDKK1 or its control. The data all came from the results of three independent experiments in this study ( ${ }^{*} \mathrm{P}<0.05,{ }^{* *} \mathrm{P}<0.01,{ }^{, * * *} \mathrm{P}<0.0001$, Student's $t$-test).

[25]. Here, we studied expression and prognosis of DKK1 in OSCC samples by analyzing the TCGA database.

Through GEPIA data analysis, we found that compared with normal oral epithelial tissues, the expression of DKK1 was significantly increased in OSCC, and it was positively correlated with the clinical staging of OSCC. Besides, the relationship between DKK1 and OSCC clinicopathological characteristics through the LinkedOmics database showed that DKK1 expression was related to pathological staging and $\mathrm{T}$ staging. At the same time, the effect of DKK1 expression level on the overall survival rate of OSCC through GEPIA found that patients with high DKK1 expression have a poorer prognosis trend. Therefore, combining the DKK1 expression status with the tumor stage is useful to predict the prognosis of OSCC.

Hence, to confirm that DKK1 served as an oncogene in OSCC, we conducted cell line verification. PCR and Western blot analysis in Figure 5 revealed that DKK1 was up-regulated in OSCC cell lines. Previous studies showed that knockdown of DKK1 suppressed the proliferation and differentiation of OSCC cells. These findings indicated that DKK1 promoted the proliferation of OSCC cells. However, it is still unclear that the mechanism of DKK1 becomes an oncogene in OSCC, but accumulating evidence reveals that upregulation of DKK1 is related to the accumulation of $\beta$-catenin. For example, Jing et al. reported that DKK1 promotes migration and invasion of non-small cell lung cancer via $\beta$-catenin signaling pathway [26]. 


\section{Conclusions}

In conclusion, we demonstrate that DKK1 is overexpressed in OSCC. Moreover, knockdown of DKK1 suppresses the cell growth of OSCC. DKK1 may play a potential therapeutic strategy for predicting the prognosis of patients in early disease stage.

\section{References}

1. Petersen PE. Oral cancer prevention and control--the approach of the World Health Organization. Oral Oncol. 2009; 45: 454-460.

2. Funk GF, Karnell LH, Robinson RA, et al. Presentation, treatment, and outcome of oral cavity cancer: a National Cancer Data Base report. Head Neck. 2002; 24: 165-180.

3. Xu Z, Jiang P and He S. Identification for Exploring Underlying Pathogenesis and Therapy Strategy of Oral Squamous Cell Carcinoma by Bioinformatics Analysis. Med Sci Monit. 2019; 25: 9216-9226.

4. Liu T, David M, Ellis O, et al. Treatment for oral squamous cell carcinoma: Impact of surgeon volume on survival. Oral Oncol. 2019; 96: 60-65.

5. Kondoh N, Mizuno-Kamiya M, Umemura N, et al. Immunomodulatory aspects in the progression and treatment of oral malignancy. Jpn Dent Sci Rev. 2019; 55: $113-120$

6. Krupnik VE, Sharp JD, Jiang C, et al. Functional and structural diversity of the human Dickkopf gene family. Gene. 1999; 238: 301-313.

7. Mao B and Niehrs C. Kremen2 modulates Dickkopf2 activity during Wnt/ LRP6 signaling. Gene. 2003; 302: 179-183.

8. Zhang F, Cao K, Du G, et al. miR-29a promotes osteoblast proliferation by downregulating DKK-1 expression and activating Wnt/beta-catenin signaling pathway. Adv Clin Exp Med. 2019; 28: 1293-1300.

9. Aguilera O, Fraga MF, Ballestar $\mathrm{E}$, et al. Epigenetic inactivation of the Wnt antagonist DICKKOPF-1 (DKK-1) gene in human colorectal cancer Oncogene. 2006; 25: 4116-4121.

10. Wang J, Shou J and Chen X. Dickkopf-1, an inhibitor of the Wnt signaling pathway, is induced by p53. Oncogene. 2000; 19: 1843-1848.

11. Liu JT, Guo WB and Sun JY. Serum Dickkopf-1 acts as a new biomarker in human breast cancer. Minerva Med. 2017; 108: 334-340.

12. Yi N, Liao QP, Li ZH, et al. RNA interference-mediated targeting of DKK1 gene expression in Ishikawa endometrial carcinoma cells causes increased tumor cell invasion and migration. Oncol Lett. 2013; 6: 756-762.

13. Mao B, Wu W, Li Y, et al. LDL-receptor-related protein 6 is a receptor for Dickkopf proteins. Nature. 2001; 411: 321-325
14. Jia X, Li N, Peng $C$, et al. miR-493 mediated DKK1 down-regulation confers proliferation, invasion and chemo-resistance in gastric cancer cells. Oncotarget. 2016; 7: 7044-7054.

15. Colla S, Zhan F, Xiong W, et al. The oxidative stress response regulates DKK1 expression through the JNK signaling cascade in multiple myeloma plasma cells. Blood. 2007; 109: 4470-4477.

16. Filipovich A, Gandhirajan RK, Gehrke I, et al. Evidence for non-functional Dickkopf-1 (DKK-1) signaling in Chronic Lymphocytic Leukemia (CLL). Eur J Haematol. 2010; 85: 309-313.

17. Thomson PJ. Perspectives on oral squamous cell carcinoma preventionproliferation, position, progression and prediction. J Oral Pathol Med. 2018; 47: 803-807.

18. Omura K. Current status of oral cancer treatment strategies: surgical treatments for oral squamous cell carcinoma. Int J Clin Oncol. 2014; 19: 423430.

19. Taghavi $\mathrm{N}$ and Yazdi I. Prognostic factors of survival rate in oral squamous cell carcinoma: clinical, histologic, genetic and molecular concepts. Arch Iran Med. 2015; 18: 314-319.

20. Igbinigie E, Guo F, Jiang SW, et al. Dkk1 involvement and its potential as a biomarker in pancreatic ductal adenocarcinoma. Clin Chim Acta. 2019; 488: 226-234.

21. Niu J, Li XM, Wang X, et al. DKK1 inhibits breast cancer cell migration and invasion through suppression of beta-catenin/MMP7 signaling pathway. Cancer Cell Int. 2019; 19: 168.

22. Lu W, Ni Z, Tong M, et al. DKK1 is epigenetically downregulated by promote methylation and inhibits bile acid-induced gastric intestinal metaplasia. Biochem Biophys Res Commun. 2020; 523: 780-786.

23. Jumpertz S, Hennes T, Asare $Y$, et al. CSN5/JAB1 suppresses the WNT inhibitor DKK1 in colorectal cancer cells. Cell Signal. 2017; 34: 38-46.

24. Zhuang $X$, Zhang $\mathrm{H}$, Li $X$, et al. Differential effects on lung and bone metastasis of breast cancer by Wnt signalling inhibitor DKK1. Nat Cell Biol. 2017; 19: 1274-1285.

25. Wang Z, Wang J, Chen Z, et al. MicroRNA-1-3p inhibits the proliferation and migration of oral squamous cell carcinoma cells by targeting DKK1. Biochem Cell Biol. 2018; 96: 355-364.

26. Zhang J, Zhang $X$, Zhao X, et al. DKK1 promotes migration and invasion of non-small cell lung cancer via beta-catenin signaling pathway. Tumour Biol. 2017; 39: 1010428317703820. 